\title{
LISA Pathfinder Performance Confirmed in an Open-Loop Configuration: Results from the Free-Fall Actuation Mode
}

M. Armano, ${ }_{1}^{1}$ H. Audley, ${ }^{2}$ J. Baird, ${ }^{3}$ P. Binetruy, ${ }^{3,}$ M. Born, ${ }^{2}$ D. Bortoluzzi, ${ }^{4}$ E. Castelli, ${ }^{5}$ A. Cavalleri, ${ }^{6}$ A. Cesarini, ${ }^{7}$ A. M. Cruise, ${ }^{8}$ K. Danzmann, ${ }^{2}$ M. de Deus Silva, ${ }^{9}$ I. Diepholz, ${ }^{2}$ G. Dixon, ${ }^{8}$ R. Dolesi, ${ }^{5}$ L. Ferraioli, ${ }^{10}$ V. Ferroni, ${ }^{5}$ E. D. Fitzsimons, ${ }^{11}$ M. Freschi, ${ }^{9}$ L. Gesa, ${ }^{12,13}$ F. Gibert, ${ }^{5,14}$ D. Giardini, ${ }^{10}$ R. Giusteri $\odot,{ }^{5,2, \dagger}$ C. Grimani, ${ }^{7}$ J. Grzymisch, ${ }^{1}$ I. Harrison, ${ }^{15}$ M-S. Hartig, ${ }^{15}$ G. Heinzel, ${ }^{2}$ M. Hewitson, ${ }^{2}$ D. Hollington, ${ }^{16}$ D. Hoyland, ${ }^{8}$ M. Hueller, ${ }^{5}$ H. Inchauspé, $, 3,17$ O. Jennrich, ${ }^{1}$ P. Jetzer, ${ }^{18}$ N. Karnesis, ${ }^{3}$ B. Kaune, ${ }^{2}$ N. Korsakova, ${ }^{19}$ C. J. Killow, ${ }^{19}$ J. A. Lobo, ${ }^{12,13,{ }^{*}}$ L. Liu, ${ }^{5}$ J. P. López-Zaragoza, ${ }^{12}$ R. Maarschalkerweerd,${ }^{15}$ D. Mance, ${ }^{10}$ N. Meshksar, ${ }^{10}$ V. Martín, ${ }^{12,13}$ L. Martin-Polo, ${ }^{9}$ J. Martino, ${ }^{3}$ F. Martin-Porqueras, ${ }^{9}$ I. Mateos, ${ }^{12,13}$ P. W. McNamara, ${ }^{1}$ J. Mendes,${ }^{15}$ L. Mendes, ${ }^{9}$ M. Nofrarias, ${ }^{12,13}$ S. Paczkowski, ${ }^{2}$ M. Perreur-Lloyd, ${ }^{19}$ A. Petiteau, ${ }^{3}$ P. Pivato, ${ }^{5}$ E. Plagnol, ${ }^{3}$ J. Ramos-Castro, ${ }^{20,13}$ J. Reiche, ${ }^{2}$ D. I. Robertson, ${ }^{19}$ F. Rivas, ${ }^{12,13}$ G. Russano, ${ }^{5,}$ J. Slutsky, ${ }^{21}$ C. F. Sopuerta, ${ }^{12,13}$ T. Sumner, ${ }^{16}$ D. Texier, ${ }^{9}$ J. I. Thorpe, ${ }^{21}$ D. Vetrugno, ${ }^{5}$ S. Vitale, ${ }^{5}$ G. Wanner, ${ }^{2}$ H. Ward ${ }^{19}$ P. J. Wass,${ }^{16,17}$ W. J. Weber, ${ }^{5}$ L. Wissel, ${ }^{2}$ A. Wittchen, ${ }^{2}$ and P. Zweifel ${ }^{10}$

${ }^{1}$ European Space Technology Centre, European Space Agency, Keplerlaan 1, 2200 AG Noordwijk, The Netherlands

${ }^{2}$ Albert-Einstein-Institut, Max-Planck-Institut für Gravitationsphysik und Leibniz Universität Hannover, Callinstraße 38, 30167 Hannover, Germany

${ }^{3} A P C$, Univ Paris Diderot, CNRS/IN2P3, CEA/lrfu, Obs de Paris, Sorbonne Paris Cité, France

${ }^{4}$ Department of Industrial Engineering, University of Trento, via Sommarive 9, 38123 Trento, and Trento Institute for Fundamental Physics and Application / INFN

${ }^{5}$ Dipartimento di Fisica, Università di Trento and Trento Institute for Fundamental Physics and Application / INFN, 38123 Povo, Trento, Italy

${ }^{6}$ Istituto di Fotonica e Nanotecnologie, CNR-Fondazione Bruno Kessler, I-38123 Povo, Trento, Italy

${ }^{7}$ DISPEA, Università di Urbino "Carlo Bo," Via S. Chiara, 2761029 Urbino/INFN, Italy

${ }^{8}$ The School of Physics and Astronomy, University of Birmingham, Birmingham, United Kingdom

${ }^{9}$ European Space Astronomy Centre, European Space Agency, Villanueva de la Cañada, 28692 Madrid, Spain

${ }^{10}$ Institut für Geophysik, ETH Zürich, Sonneggstrasse 5, CH-8092, Zürich, Switzerland

${ }^{11}$ The UK Astronomy Technology Centre, Royal Observatory, Edinburgh, Blackford Hill, Edinburgh, EH9 3HJ, United Kingdom

${ }^{12}$ Institut de Ciències de l'Espai (ICE, CSIC), Campus UAB, Carrer de Can Magrans s/n, 08193 Cerdanyola del Vallès, Spain

${ }^{13}$ Institut d'Estudis Espacials de Catalunya (IEEC), C/ Gran Capità 2-4, 08034 Barcelona, Spain

${ }^{14}$ isardSAT SL, Marie Curie 8-14, 08042 Barcelona, Catalonia, Spain

${ }^{15}$ European Space Operations Centre, European Space Agency, 64293 Darmstadt, Germany

${ }^{16}$ High Energy Physics Group, Physics Department, Imperial College London, Blackett Laboratory, Prince Consort Road, London, SW7 2BW, United Kingdom

${ }^{17}$ Department of Mechanical and Aerospace Engineering, MAE-A, P.O. Box 116250, University of Florida, Gainesville, Florida 32611, USA

${ }^{18}$ Physik Institut, Universität Zürich, Winterthurerstrasse 190, CH-8057 Zürich, Switzerland

${ }^{19}$ SUPA, Institute for Gravitational Research, School of Physics and Astronomy, University of Glasgow, Glasgow, G12 8QQ, United Kingdom

${ }^{20}$ Department d'Enginyeria Electrònica, Universitat Politècnica de Catalunya, 08034 Barcelona, Spain

${ }^{21}$ Gravitational Astrophysics Lab, NASA Goddard Space Flight Center, 8800 Greenbelt Road, Greenbelt, Maryland 20771, USA

(Received 19 February 2019; revised manuscript received 11 June 2019; published 11 September 2019)

We report on the results of the LISA Pathfinder (LPF) free-fall mode experiment, in which the control force needed to compensate the quasistatic differential force acting on two test masses is applied intermittently as a series of "impulse" forces lasting a few seconds and separated by roughly $350 \mathrm{~s}$ periods of true free fall. This represents an alternative to the normal LPF mode of operation in which this balancing force is applied continuously, with the advantage that the acceleration noise during free fall is measured in the absence of the actuation force, thus eliminating associated noise and force calibration errors. The differential acceleration noise measurement presented here with the free-fall mode agrees with noise measured with the continuous actuation scheme, representing an important and independent confirmation

Published by the American Physical Society under the terms of the Creative Commons Attribution 4.0 International license. Further distribution of this work must maintain attribution to the author(s) and the published article's title, journal citation, and DOI. 
of the LPF result. An additional measurement with larger actuation forces also shows that the technique can be used to eliminate actuation noise when this is a dominant factor.

DOI: 10.1103/PhysRevLett.123.111101

Introduction.-LISA Pathfinder (LPF) [1] was a differential accelerometer designed to demonstrate the free fall of geodesic reference test masses (TMs) at the level required for space-borne gravitational wave observatories such as LISA [2]. LPF achieved this by using a high precision interferometer to measure the relative acceleration, $\Delta g$, between two TMs placed in the same spacecraft (SC), along the $x$ axis joining their centers (see Fig. 1). LISA is a truly open-loop differential acceleration measurement, with both TMs unforced inside separate drag-free spacecrafts. In LPF, closed-loop forces must be employed to keep the two TMs inside a single spacecraft, and this applied force is part of the measurement. Indeed, it is not possible for both TMs to be in free fall along $x$ at the same time, like would be in LISA.

In the normal LPF operations conditions, the observable $\Delta g$ is measured by applying a calibrated compensation force $g_{c}$ on TM2 (all forces are expressed here per unit mass) and is extracted according to $\Delta g \simeq \Delta \ddot{x}-g_{c}$, with $\Delta \ddot{x}$ being the numerical second time derivative of the relative displacement $\Delta x$. The compensation is exerted by an electrostatic control loop continuously acting on TM2 with unity gain around $1 \mathrm{mHz}$. The reconstructed signal for $\Delta g$ is dominated by $g_{c}$ for frequencies roughly below the $1 \mathrm{mHz}$ band of the controller, while $\Delta \ddot{x}$ leads at higher frequencies where the TM is essentially free. The resulting time series for $\Delta g$ depends on the actuator calibration [3]. In addition, the voltage noise of the actuator that applies $g_{c}$ introduces an extra force noise that was expected to be dominant at low frequencies [4].

To measure acceleration noise in a LISA-like configuration without $x$ axis applied forces, a dedicated noise measurement using intermittent free fall has been designed

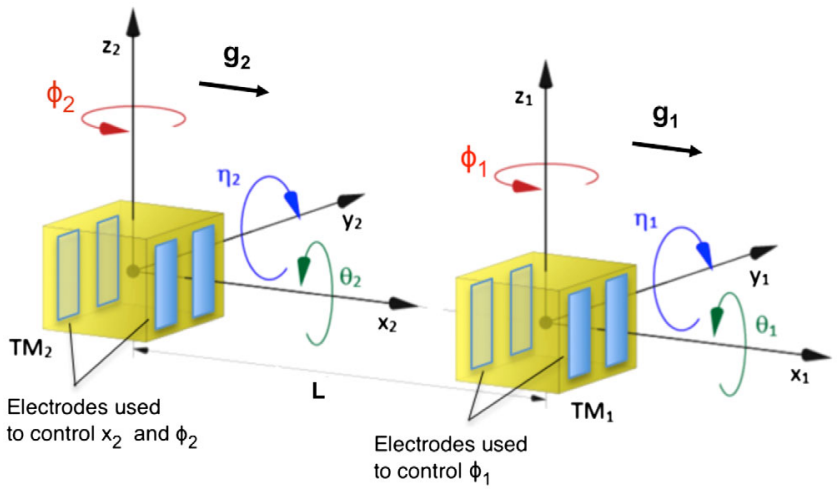

FIG. 1. LPF capacitive actuation along $x$ and housing coordinate systems. $g_{1}$ and $g_{2}$ indicate the stray acceleration experienced by TM1 and TM2, respectively.
[5]. This alternative technique aims at estimating the residual noise in $\Delta g$ independent of the actuator calibration and free of actuation noise, and to characterize, by comparison, the contribution of actuation noise measured in standard operations. This configuration was tested in the LPF free-fall mode (or "drift" mode) experiment in which the compensation force on TM2 is applied intermittently in the form of high amplitude pulses with period of a few seconds, in between which the TM is let to fly with no compensation force along $x$.

The free-fall mode provides a measurement of the noise in $\Delta g$ that coincides with that measured in the standard LPF configuration with continuous control and thus it confirms, as an independent measurement, the LPF performance. Indeed, actuation noise measured in flight conditions was a not a dominant contributor around $1 \mathrm{mHz}$, thus removing the $x$ actuator produced a small effect on the acceleration noise spectrum. Moreover, the presented result demonstrates the functionality of an alternative control for spacebased gradiometers [6], where force gradients are measured from the applied compensation force needed to hold the TM steady.

LISA Pathfinder instrument.-Two gold-platinum cubic test-masses separated by $\sim 38 \mathrm{~cm}$ form the core instrument of LPF [7]. Both are in free fall inside a single SC with no mechanical contact and each of them is contained within an electrode housing [8], which serves as a 6 degree-offreedom capacitive sensor and electrostatic force and torque actuator. TM2 is forced by an electrostatic suspension control loop to stay at a fixed distance from TM1, along $x$ and thus centered in its own electrode housing. A second controller, called drag free, feeds the thrusters to keep the SC to follow TM1.

Given the quadratic dependence of force on voltage, the force fluctuation associated with an actuation voltage amplitude fluctuation depends on the force levels applied by each electrode. The same four electrodes actuate in $x$ and $\phi$ (see Fig. 1), with an actuation scheme that keeps the stiffness constant [4] according to the maximum net forces and torques allowed, called "authorities." The resulting $x$ force noise from actuation amplitude fluctuations depends both on the net applied $x / \phi$ force and on the $x / \phi$ authorities (the actuation scheme and noise model are presented in an upcoming publication).

Based on a preflight analysis that considered $\Delta g_{\mathrm{dc}}=650 \mathrm{pm} / \mathrm{s}^{2}$-based on conservative gravitational balance precision estimates [9] and measured actuation amplitudes between 3 and $8 \mathrm{ppm} / \mathrm{Hz}^{1 / 2}$ —actuation amplitude fluctuations were considered as the leading low frequency acceleration noise source for LPF at roughly 
TABLE I. Free-fall mode measurement data set. The table includes the authority levels, duration ( $\Delta t$, in hours), initial flight amplitude $\left(\Delta x_{0}\right)$, and TM2 stiffness $\left(\omega_{2}^{2}\right)$. In both cases $\omega_{2}^{2}$ is in agreement $(1 \sigma)$ with the values resulting from the system dynamics calibration presented in Ref. [12]. The force authority values in bracket refer to the continuous control.

\begin{tabular}{lccccccr}
\hline \hline Authority scheme & $g_{x_{2}}^{\max }$ & $g_{\phi_{1}}^{\max }$ & $g_{\phi_{2}}^{\max }$ & Start date & $\Delta t$ & $\Delta x_{0}$ & $\omega_{2}^{2}$ \\
\cline { 2 - 8 } & {$\left[\mathrm{pm} / \mathrm{s}^{2}\right]$} & {$\left[\mathrm{nrad} / \mathrm{s}^{2}\right]$} & {$\left[\mathrm{nrad} / \mathrm{s}^{2}\right]$} & $(2016)$ & {$[\mathrm{h}]$} & {$[\mathrm{nm}]$} & {$\left[1 / \mathrm{s}^{2} \times 10^{-7}\right]$} \\
\hline Nominal & $0(1100)$ & 15 & 15 & $09 / 06$ & 18 & $\sim 40$ & $-7.12 \pm 0.03$ \\
URLA & $0(26)$ & 2.2 & 1.4 & $18 / 12$ & 132 & $\sim 30$ & $-4.53 \pm 0.09$ \\
\hline \hline
\end{tabular}

$7 \mathrm{fm} / \mathrm{s}^{2} / \mathrm{Hz}^{1 / 2}$ at $1 \mathrm{mHz}$ [4] (this analysis considered $\Delta g_{\mathrm{dc}} \approx 650 \mathrm{~nm} / \mathrm{s}^{2}$ and $\phi$ dc angular accelerations of $2 \mathrm{nrad} / \mathrm{s}^{2}$, with $10 \%$ larger actuation authorities to accommodate transient dynamics). Over the mission, different levels of force and torque authority were implemented, beginning with the nominal configuration programmed before flight to accommodate potentially large gravitational imbalances, with $x$-force authority of $1100 \mathrm{pm} / \mathrm{s}^{2}$ (see Table I). The in-flight observed dc force imbalance was much smaller, always below $20 \mathrm{pm} / \mathrm{s}^{2}$ along $x$ [3] with angular accelerations of -1.1 and $0.2 \mathrm{nrad} / \mathrm{s}^{2}$ for TM1 and TM2. This allowed reducing the authorities from nominal to the URLA configuration levels, with $26 \mathrm{pm} / \mathrm{s}^{2} x$-force authority (see Table I). In this configuration, used for the measurements that established the published LPF differential acceleration noise floor [3,10], the actuation noise, as estimated from a dedicated in-flight measurement campaign employing various force levels, is less than $20 \%$ of the total acceleration noise power measured over the 0.1 to $1 \mathrm{mHz}$ band [11] (see the dashed line in Fig. 4).

Removing the $x$-axis actuation with the free-fall mode thus, in these flight conditions, is expected to have only a small impact on the measured acceleration noise (we note that during the free-fall mode the $\phi$ actuation torque is still applied continuously). Nevertheless, the free-fall mode experiment still represents an independent measurement of the differential acceleration without any actuator, immune to possible actuation nonlinearities or calibration inaccuracies.

Experiment description and calibration.-The free-fall mode implemented on LPF is a special actuation scheme where the electrostatic control on TM2 is switched on the sensitive $x$ axis only for a very short duration $(\leq 5 \mathrm{~s})$. In particular, an impulse controller tracks the TM2 displacement, $x_{2}$, during the flight and estimates the impulse necessary to push it back on the other side against the static field it experiences on board the SC. Then, the impulse-flight cycle is repeated (see Fig. 2) [5,13]. The flight interval, $T_{\text {flight }}$, is set by the maximum displacement allowed along $x_{2}(\approx 10 \mu \mathrm{m})$, based on the preflight estimate of the gravitational imbalance. The experiments presented here are implemented with a fixed experimental time, $T_{\text {exp }}=T_{\text {flight }}+T_{\text {imp }}$, of $350.2 \mathrm{~s}$, while impulse durations $\left(T_{\mathrm{imp}}\right)$ of $1 \mathrm{~s}$ and $5 \mathrm{~s}$ were used in the two measurements.
Figure 2 depicts the start of the first experiment with freefall mode performed with $T_{\mathrm{imp}}=1 \mathrm{~s}$ and following a noise run executed in continuous control mode. As visible in the middle panel, the free fall mode is characterized by a wide dynamic range in displacement (tens of $\mathrm{nm}$ ), in contrast with the continuous mode (tens of pm [13]).

The main observable of LPF, $\Delta g$, is calculated with freefall data as follows:

$$
\Delta g(t) \equiv \Delta \ddot{x}(t)+\omega_{2}^{2} \Delta x(t)-g_{\mathrm{rot}}(t)
$$

where $\omega_{2}^{2}$ is the electrostatic force gradient ("stiffness"), coupling TM2 to the SC and $g_{\text {rot }}(t)$ is the contribution of the inertial forces acting on the TMs which are described and calculated in Ref. [12]. Differently from the definition of $\Delta g$ in Ref. [10], the control force on TM2 is excluded in Eq. (1), being zero by definition in free-fall mode.

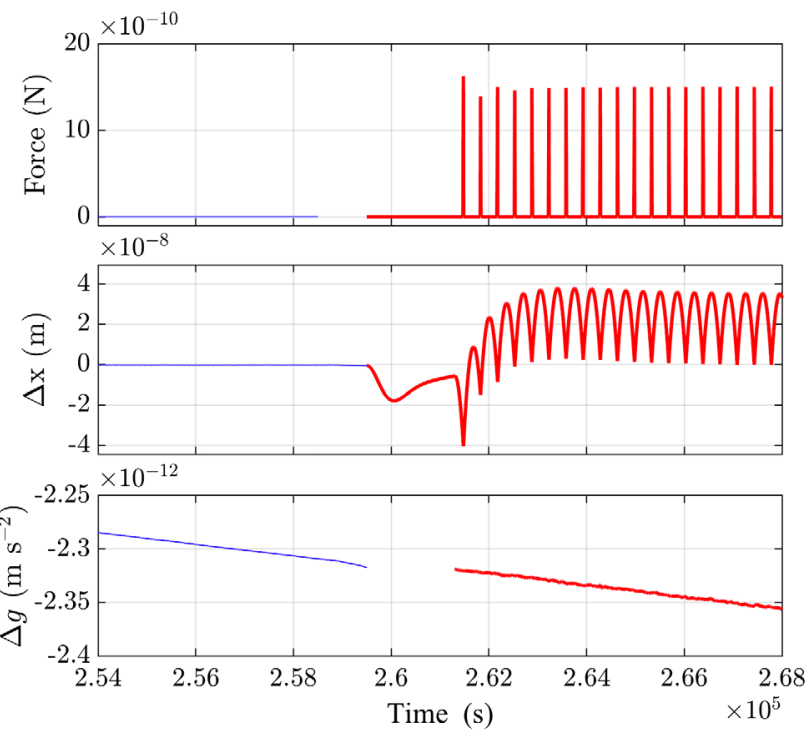

FIG. 2. Time series of TM2 force (top), relative displacement, $\Delta x$ (middle), and $\Delta g$ (bottom) measured in June 2016. The thin lines refer to a noise measurement with continuous control on TM2 in URLA authority, while the thick lines indicate a freefall mode measurement in nominal authority. The discontinuity in the top panel stems from the use of different telemetry packets. The transient phase between the two runs is discarded in the bottom panel. 
In addition, the differential stiffness coupling the SC motion to $\Delta g$ is neglected in our analysis, as it is too small to impact the result.

To retrieve the stiffness on TM2, $\omega_{2}^{2}$, we fit $\Delta \ddot{x}$ to $-\omega_{2}^{2} \Delta x$ flight by flight, as described in Ref. [13]. The resulting parameter values are averaged over the flights to get a single estimate. Then, the inertial contribution is subtracted from the residuals of the fit [see Eq. (1)] according to the procedure explained in Ref. [12].

Measurement data set.-The free-fall mode experiment was performed seven times between June and December 2016, with stable and reliable control operation in various actuation configurations. This Letter presents the one-day measurement executed in June with $\phi$ authority based on preflight analyses (nominal authority) and the last run, with one week duration, performed in December with lower authority levels on $\phi$ (URLA authority). The intermediate measurements were used for planning the last long run, which was implemented to limit the flight amplitude within tens of $\mathrm{nm}$. Indeed, the large dynamic range achieved in free-fall mode, compared to the continuous control mode, impacts the interferometer readout. In addition, it increases timing error issues, as observed also in the dedicated onground testing campaign performed with a torsion pendulum facility [14]. In this context, to reduce the gravitational imbalance between the TMs measured in December, TM1 was actuated along $x$ with a constant out of loop force with amplitude of $11.2 \mathrm{pN}$, which was then subtracted from $\Delta g$. It has been verified that this force does not introduce significant noise or calibration errors.

Table I reports details and calibration results of the two free-fall mode measurements presented here.

Data analysis.-The analysis of the free-fall mode experiment is challenging due to the presence of impulses. Estimating the noise in $\Delta g$ without actuation implies limiting the analysis to the free-fall periods alone, effectively "gapping" data to be insensitive to the noise from the high-force impulses. The effect of gaps on the spectrum must be characterized, especially at low frequency, where the noise is expected to be lower than in presence of control [11].

In general, gaps can corrupt the spectral estimation, in the form of spectral leakage from both high and low frequencies, thus introducing a systematic bias in the underlying spectrum. Gaps can be masked with smooth spectral windows or filled with synthetic noise. In this Letter, we present the results obtained by applying the "Blackman-Harris gap zero" (BHGZ) technique (see Ref. [14] for a full review, except for the bias removal). The method, implemented using the dedicated data analysis toolbox, LTPDA [15], consists in filling the gaps with zero numerically by means of a rectangular-wave window, after having low-pass filtered and decimated the $\Delta g$ time series. The name of the approach refers to the shape of the filter chosen, that is a minimum 4-term Blackman-Harris (BH) window. The filter is applied to reduce the aliasing caused by the rectangular-wave window and it is a finite impulse response (FIR) filter to avoid mixing in the gaps. Indeed, compared to smoother windows, the rectangular-wave window produces a relevant spectral leakage of the noise, from high frequency into the low frequency band of the spectrum. Finally, the downsampling is imposed by the numerical limitation of the procedure applied to remove, from the spectrum, the remaining bias due to gaps. This procedure will be described below, while implementation details of the BHGZ technique are found in Refs. $[13,16]$.

The PSD of filtered, decimated, and gapped data is estimated with the same technique as for the continuous data described in Ref. [3], with errors estimation based on $\chi^{2}$ statistics [10]. Then, it is normalized for the transfer function of the $\mathrm{BH}$ filter and finally corrected for the bias induced by gaps.

The spectral bias in free-fall data appears in the form of peaks at harmonics of the gap frequency $\left(\equiv 1 / T_{\exp } \sim 2.8 \mathrm{mHz}\right.$, see Fig. 3), observed after the multiplication of data by the rectangular-wave window. In addition, the amplitude of the gapped spectrum is reduced compared to that of continuous data, due to removal of data points, as reported e.g., in Ref. [20] and discussed also in the Supplemental Material [16], and this reduction scales with the gap size. In particular, in case of white noise, the normalization factor needed to compensate for the missing points set to zero, is equal to the inverse of the rectangular-wave duty cycle, as demonstrated in Ref. [14].

To remove the bias we follow "a pseudo-inverse" approach, described in detail in the Supplemental Material [16], based on looking for the theoretical shape of the spectrum that, through the action of the rectangularwave window, reproduces the experimental spectrum. In practice, we fit the gapped spectrum to a smooth continuous model, we assume underneath data, which is convolved

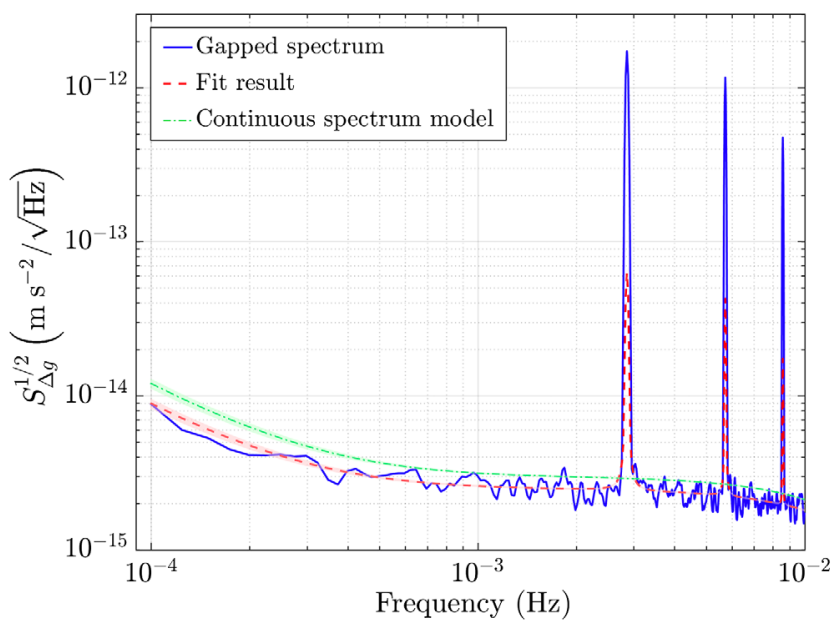

FIG. 3. Fit results to free-fall data, measured in December 2016, to remove the bias from the spectrum (see the text for details). The peaks are excluded from the analysis and hence not fitted. 
with the rectangular-wave window. In our case, the lowfrequency noise only is modeled and the fit is performed at samples away from the peaks which we do not model. Indeed, we are mainly interested in removing the bias at low frequencies in order to estimate the noise in absence of the compensation force on TM2. The noise model, reported in Eq. (7) in the Supplemental Material [16], is based on the measured noise with continuous actuation [10] and it is precise enough as we achieve a good quality of fit [see, as an example, Fig. 2(b) in the Supplemental Material [16]].

The fit parameters are then used to trace the "native" spectrum of free-fall mode data without gaps, as explained in the Supplemental Material [16]. Figure 3 shows the result, in terms of $\Delta g$ ASD (amplitude spectral density), of this procedure on data of the free-fall mode experiment carried out in December. The result obtained from the best fit to the ASD of data (solid line) is indicated by the dashed line, while the dash-dotted line is the model for the underlying continuous differential acceleration noise spectrum, resulting from our analysis, which converts into the dashed line when gaps are inserted. Thus, the bias is removed from the experimental gapped spectrum (solid line), by multiplying it by the ratio between the dash-dotted and the dashed lines. The effective experimental curve, with points appropriately scaled by the ratio of the dashdotted and dashed lines, is shown by the dot data points in Fig. 4. Details of the analysis of December data can be found in the Supplemental Material [16].

Applying the technique on continuous control $\Delta g$ data, with artificially inserted gaps, accurately recovers the spectrum obtained when analyzing the full continuous data

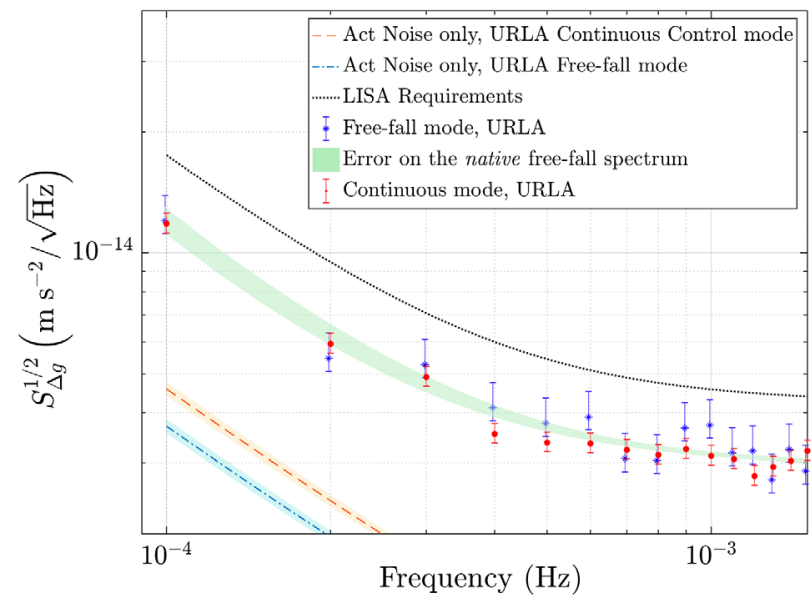

FIG. 4. Acceleration noise estimate with free-fall mode in URLA $\phi$ authority compared with that in continuous actuation mode in URLA and the LISA requirements [2]. The free-fall ASD (dots) results from 20 periodograms, while the continuous noise run (asterisks) is $\sim 18$ days long (78 periodograms), both calculated at $1 \sigma$ confidence interval and according to the method presented in [10]. Shadowed area: estimate of the "native" freefall spectrum. Dashed lines: actuation noise predictions. set. The results of the method calibration are reported in the Supplemental Material [16].

Results._URLA authority: Figure 4 shows the $\Delta g$ ASD of the free-fall mode experiment performed with URLA $\phi$ authority (asterisk data points), compared with that measured with continuous control mode in the same authority and just after the free-fall mode experiment (dot data points). Figure 4 includes the actuation noise predictions in URLA authority for both the measurements [11,13], showing that actuation noise does not dominate the low frequency spectrum in URLA continuous control mode and that it is expected to lessen, in free-fall mode, by roughly $20 \%$ at $0.1 \mathrm{mHz}$ in ASD. The shadowed area behind the data points coincides with that of the dash-dotted line of Fig. 3. As visible, at frequencies below $1 \mathrm{mHz}$ the $\Delta g$ estimate in URLA free-fall mode agrees, within $1 \sigma$, with that measured in continuous control. Thus, removing the $x$ control does not significantly reduce noise along the sensitive axis, since actuation noise in continuous mode is already dominated by the $\phi$ control, which does not change in free-fall mode.

While the noise reduction is not resolvable, the free-fall mode result represents an important confirmation of the LPF differential acceleration benchmark without applied forces. It also confirms that the low frequency noise excess, visible around $0.1 \mathrm{mHz}$ and currently under investigation [10], is not caused by inaccuracies in the $x$-force subtraction, as the free-fall mode completely removes such contribution: we can state that noise from possible errors in the $x$-actuator calibration is below our detection threshold. To conclude, the free-fall mode experiment in the low $\phi$ authority confirms, as an independent measurement, the LPF performance achieved in continuous control mode.

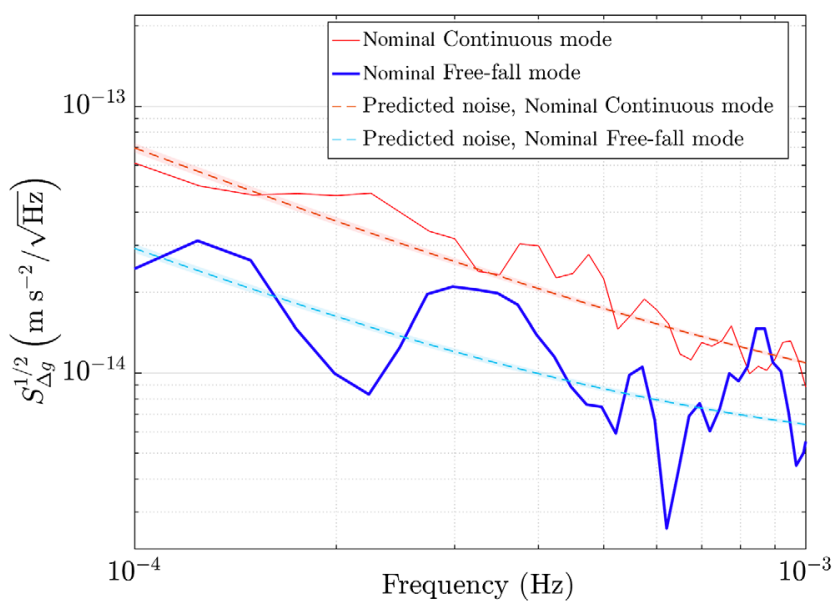

FIG. 5. Comparison of $\Delta g$ ASD between the free-fall mode experiment executed in June 2016 in nominal $\phi$ authority (thick solid line, 2 periodograms) and the continuous control mode run carried out in May 2016 in the same authority (thin solid line, 3 periodograms). The dashed lines are the noise predictions as explained in the text. 
Nominal authority: The results of the one-day experiment executed with free-fall mode in nominal $\phi$ authority, is depicted in Fig. 5 (thick solid line). The thin solid line indicates a $\Delta g$ estimate measured in the period of the freefall run with nominal continuous control. The picture includes the expected low-frequency noise at that period of time for both the measurements (dashed lines) [11]. As visible, in this case turning off the nominal authority $\left(\sim 1100 \mathrm{pm} / \mathrm{s}^{2}\right) x$ actuator, reduces noise at low frequency effectively, matching the predictions of suppression of actuation noise along the sensitive axis, which in turn dominates the spectrum when active. The free-fall mode thus can be considered an alternative technique to eliminate actuation noise when this is a limiting factor.

To conclude, though the noise due to the $x$ control does not dominate the low-frequency band in the low authority scheme, as confirmed by the free-fall mode results, actuation noise enters in the LISA noise budget through the $\phi$ control. In this context, the free-fall mode experiment has provided an acceleration noise measurement in an actuation configuration similar to that of LISA.

This work has been made possible by the LISA Pathfinder mission, which is part of the space-science program of the European Space Agency. The French contribution has been supported by CNES (Accord Specific de projet CNES 1316634/CNRS 103747), the CNRS, the Observatoire de Paris and the University ParisDiderot. E. Plagnol and H. Inchauspé would also like to acknowledge the financial support of the UnivEarthS Labex program at Sorbonne Paris Cité (ANR-10-LABX0023 and ANR-11-IDEX-0005-02). The Albert-EinsteinInstitut acknowledges the support of the German Space Agency, DLR. The work is supported by the Federal Ministry for Economic Affairs and Energy based on a resolution of the German Bundestag (FKZ 50OQ0501 and FKZ 50OQ1601). The Italian contribution has been supported by Agenzia Spaziale Italiana and Istituto Nazionale di Fisica Nucleare. The Spanish contribution has been supported by Contracts No. AYA2010-15709 (MICINN), No. ESP2013-47637-P, and No. ESP2015-67234-P and No. ESP2017-90084-P (MINECO), and 2017-SGR-1469 (AGAUR). M. Nofrarias acknowledges support from Fundacion General CSIC (Programa ComFuturo). F. Rivas acknowledges support from a Formación de Personal Investigador (MINECO) contract. The Swiss contribution acknowledges the support of the Swiss Space Office (SSO) via the PRODEX Programme of ESA. L.F. acknowledges the support of the Swiss National Science Foundation Project Number 200021162449. The UK groups wish to acknowledge support from the United Kingdom Space Agency (UKSA), the University of Glasgow, the University of Birmingham, Imperial College, and the Scottish Universities Physics Alliance (SUPA). J. I. Thorpe and J. Slutsky acknowledge the support of the U.S. National Aeronautics and Space Administration (NASA).
*Deceased.

†roberta.giusteri@aei.mpg.de

giuliana.russano@unitn.it

[1] P. McNamara, S. Vitale, K. Danzmann (LISA Pathfinder Science Working Team), LISA Pathfinder, Classical Quantum Gravity 25, 114034 (2008).

[2] P. Amaro-Seoane et al., Laser interferometer space antenna, arXiv: 1702.00786.

[3] M. Armano et al., Sub-Femto- $g$ Free Fall for Space-Based Gravitational Wave Observatories: LISA Pathfinder Results, Phys. Rev. Lett. 116, 231101 (2016).

[4] F. Antonucci et al., From laboratory experiments to LISA Pathfinder: Achieving LISA geodesic motion, Classical Quantum Gravity 28, 094002 (2011).

[5] A. Grynagier, W. Fichter, and S. Vitale, The LISA Pathfinder drift mode: Implementation solutions for a robust algorithm, Classical Quantum Gravity 26, 094007 (2009).

[6] R. Rummel, W. Yi, and C. Stummer, GOCE gravitational gradiometry, J. Geod. 85, 777 (2011).

[7] L. Carbone, A. Cavalleri, R. Dolesi, C. D. Hoyle, M. Hueller, S. Vitale, and W. J. Weber, Characterization of disturbance sources for LISA: Torsion pendulum results, Classical Quantum Gravity 22, S509 (2005).

[8] R. Dolesi, D. Bortoluzzi, P. Bosetti, L. Carbone, A. Cavalleri, I. Cristofolini, M. DaLio, G. Fontana, V. Fontanari, B. Foulon, C. D. Hoyle, M. Hueller, F. Nappo, P. Sarra, D. N. A. Shaul, T. Sumner, W. J. Weber, and S. Vitale, Gravitational sensor for LISA and its technology demonstration mission, Classical Quantum Gravity 20, S99 (2003).

[9] M. Armano et al., Constraints on LISA Pathfinder's selfgravity: Design requirements, estimates and testing procedures, Classical Quantum Gravity 33, 235015 (2016).

[10] M. Armano et al., Beyond the Required LISA Free-Fall Performance: New LISA Pathfinder Results down to $20 \mu \mathrm{Hz}$, Phys. Rev. Lett. 120, 061101 (2018).

[11] W. J. Weber et al. (to be published).

[12] M. Armano et al., Calibrating the system dynamics of LISA Pathfinder, Phys. Rev. D 97, 122002 (2018).

[13] R. Giusteri et al., The free-fall mode experiment on LISA Pathfinder: First results, J. Phys. Conf. Ser. 840, 012005 (2017).

[14] G. Russano, A. Cavalleri, A. Cesarini, R. Dolesi, V. Ferroni, F. Gibert, R. Giusteri, M. Hueller, L. Liu, P. Pivato, H. B. Tu, D. Vetrugno, S. Vitale, and W. J. Weber, Measuring fN force variations in the presence of constant $\mathrm{nN}$ forces: a torsion pendulum ground test of the LISA Pathfinder free-fall mode, Classical Quantum Gravity 35, 035017 (2018).

[15] M. Hewitson et al., Classical and quantum gravity data analysis for the lisa technology package, Classical Quantum Gravity 26, 094003 (2009).

[16] See Supplemental Material at http://link.aps.org/ supplemental/10.1103/PhysRevLett.123.111101, sections "BHGZ method implementation" for implementation details of the BHGZ technique, "Final considerations" for discussion about the amplitude of the spectrum peaks, "Bias removal algorithm" for details on the bias removal algorithm, "Bias removal on free-fall mode data measured in December" for the bias removal procedure applied on data of free-fall mode, "Calibration of the method with 
continuous noise data" for the bias removal procedure applied on continuous data for calibration purposes, which includes Refs. [10,14,17-20].

[17] S. Vitale et al., Data series subtraction with unknown and unmodeled background noise, Phys. Rev. D 90, 042003 (2014).

[18] L. Ferraioli, G. Congedo, M. Hueller, S. Vitale, M. Hewitson, M. Nofrarias, and M. Armano, Quantitative analysis of LISA pathfinder test-mass noise, Phys. Rev. D 84, 122003 (2011).
[19] P. Welch, The use of fast Fourier transform for the estimation of power spectra: A method based on time averaging over short, modified periodograms, IEEE Trans. Audio Electroacoust. 15, 70 (1967).

[20] Q. Baghi, G. Métris, J. Bergé, B. Christophe, P. Touboul, and M. Rodrigues, Regression analysis with missing data and unknown colored noise: Application to the MICROSCOPE space mission, Phys. Rev. D 91, 062003 (2015). 